\title{
Hemodynamics model of fluid-solid interaction in internal carotid artery aneurysms
}

\author{
Xu Bai-Nan • Wang Fu-Yu • Liu Lei • Zhang Xiao-Jun • \\ Ju Hai-Yue
}

Received: 19 December 2009/Revised: 17 May 2010 /Accepted: 5 July 2010 / Published online: 2 September 2010

(C) The Author(s) 2010. This article is published with open access at Springerlink.com

\begin{abstract}
The objective of this study is to present a relatively simple method to reconstruct cerebral aneurysms as 3D numerical grids. The method accurately duplicates the geometry to provide computer simulations of the blood flow. Initial images were obtained by using CT angiography and 3D digital subtraction angiography in DICOM format. The image was processed by using MIMICS software, and the 3D fluid model (blood flow) and 3D solid model (wall) were generated. The subsequent output was exported to the ANSYS workbench software to generate the volumetric mesh for further hemodynamic study. The fluid model was defined and simulated in CFX software while the solid model was calculated in ANSYS software. The force data calculated firstly in the CFX software were transferred to the ANSYS software, and after receiving the force data, total mesh displacement data were calculated in the ANSYS software. Then, the mesh displacement data were transferred back to the CFX software. The data exchange was processed in workbench software. The results of simulation could be visualized in CFX-post. Two examples of grid reconstruction and blood flow simulation for patients with internal carotid artery aneurysms were presented. The wall shear stress, wall
\end{abstract}

X. Bai-Nan $(\bowtie) \cdot$ W. Fu-Yu $\cdot$ L. Lei

Department of Neurosurgery, Chinese PLA General Hospital,

Beijing 100853, China

e-mail: shjwkk@sina.com

Z. Xiao-Jun

College of Mechanical Engineering and Applied Electronics

Technology, Beijing University of Technology,

Beijing 100124, China

J. Hai-Yue

Department of Radiology, Chinese PLA General Hospital,

Beijing 100853, China total pressure, and von Mises stress could be visualized. This method seems to be relatively simple and suitable for direct use by neurosurgeons or neuroradiologists, and maybe a practical tool for planning treatment and follow-up of patients after neurosurgical or endovascular interventions with 3D angiography.

Keywords Hemodynamics · Fluid-solid interaction (FSI) . Aneurysms · Computational fluid dynamic (CFD)

\section{Introduction}

The flow dynamics plays an important role in the initiation, growth, and rupture of cerebral aneurysms [12, 17]. Because of technical factors, most works related to the intra-aneurysmal hemodynamics have largely focused on idealized aneurysm geometry or surgically created aneurysms in animals. In vitro studies have allowed a very detailed measurement of hemodynamic variables but are of limited value in understanding the hemodynamic forces in an individual clinical case. Current technology cannot provide analogous information in vivo through either invasive or noninvasive measurements. Wall shear stress (WSS) is a flow-induced stress that can be described as the frictional force of viscous blood. According to the mechanics of the materials, the maximum shear stress safety tool is based on the maximum shear stress failure theory for ductile materials. The theory states that a particular combination of principal WSSs causes failure if the maximum shear equals or exceeds a specific shear limit. Furthermore, von Mises stress (VMS, also called equivalent stress) is often used in design work because it allows any arbitrary 3D stress state to be represented as a single positive stress value. Equivalent stress is part of the 
maximum equivalent stress failure theory used to predict yielding in a ductile material.

The direct stress measurements in cerebral aneurysmal patients are not available. Though in vivo measurements of the $3 \mathrm{D}$ velocity field and WSS have become possible with the development of phase-contrast magnetic resonance velocimetry for large and simple arteries like the aorta or the carotid bifurcation, phase-contrast magnetic resonance velocimetry cannot be applied in calculating the spatial distribution of the WSS in small and tortuous vessels like the intracranial arteries. The other clinical technique for assessing flow velocities, specifically sonography, is unable to provide access to flow values in the basilar artery or in the vertebral arteries immediately proximal to their junction. Therefore, researchers have turned to computational fluid dynamic (CFD) simulations to gain a better understanding of the intraaneurysmal hemodynamics.

Computational-based models provide an attractive method of investigating intra-aneurysmal flow dynamics by providing the ability to theoretically model and study any possible geometry $[7,16]$. Until recently, most computational studies have been performed on the specific patient geometry and to connect specific hemodynamic factors to clinical events allowing statistical analysis in a patient population [2]. These analyses have been done on a variety of assumptions used in modeling including laminar flow, Newtonian properties of fluids, and rigid wall. Actually, the wall of vessels and aneurysms were elastic; effect of pulsatile motion of the wall may be important for the accurate determination of intra-aneurysmal flow patterns, and the material failure rates in CFD models incorporating the mechanical properties of walls may also be important for the rupture of aneurysms, so the fluid-solid interaction (FSI) should be considered. In this work, we describe the method to analyze the complex interaction of blood flow and the compliant aneurysmal wall, so the results of intraaneurysmal hemodynamics were more realistic.

\section{Mathematical model}

Fluid model

The blood flow was assumed to be laminar and Newtonian. The incompressible Navier-Stokes equations were used as the governing equations which are suitable for problems with FSI.

$$
\begin{aligned}
& \nabla \cdot u=0 \\
& \partial u / \partial t+\left(u-u_{\mathrm{g}}\right) \cdot \nabla u=-\nabla p / \rho_{\mathrm{f}}+v \Delta u
\end{aligned}
$$

where $\rho_{\mathrm{f}}$ is the fluid density, $u$ is the fluid velocity vector and is the moving coordinate velocity, respectively. In the
Arbitrary Lagrangian Eulerian (ALE) formulation [18], $u-u_{\mathrm{g}}$ is the relative velocity of the fluid with respect to the moving coordinate velocity, and $\tau$ is the deviatoric stress tensor. Blood is modeled to have a density $\rho_{\mathrm{f}}=1,053 \mathrm{~kg} / \mathrm{m}^{3}$.

Solid model

The governing equation for the solid domain is the momentum conservation equation given by Eq. 2. In contrast to the ALE formulation of the fluid equations, a Lagrangian coordinate system is adopted [18]:

$\nabla \sigma_{\mathrm{s}}=\rho_{\mathrm{s}} \ddot{d}_{\mathrm{s}}$

where $\rho_{s}$ is the solid density, $\sigma_{s}$ is the solid stress tensor, and $\ddot{d}_{\mathrm{s}}$ is the local acceleration of the solid. The artery wall was assumed to be hyperelastic, isotropic, incompressible, and homogeneous with a density $\rho_{\mathrm{s}}=1,050 \mathrm{~kg} / \mathrm{m}^{3}$.

Fluid-structure interaction model

The boundary conditions on the FSI interfaces state that (1) displacements of the fluid and solid domain must be compatible, (2) tractions at these boundaries must be at equilibrium, and (3) fluid obeys the no-slip condition. These conditions are given in Eq. 3:

$$
\begin{aligned}
d_{\mathrm{s}} & =d_{\mathrm{f}} \\
\sigma_{\mathrm{s}} \cdot \widehat{n}_{\mathrm{s}} & =\sigma_{\mathrm{f}} \cdot \widehat{n}_{\mathrm{f}} \\
\dot{d}_{\mathrm{s}} & =\dot{d}_{\mathrm{f}}
\end{aligned}
$$

where $d, \sigma$, and $\widehat{n}$ are displacements, stress tensors, and boundary normals with the subscripts $\mathrm{f}$ and $\mathrm{s}$ indicating a property of the fluid and solid, respectively. The 3D hyperelastic M-R model was used to describe the material properties of the artery wall [18].

\section{Numerical methods}

\section{CT angiography}

CT angiography (CTA) (Fig. 1) was done by using a helical CT scanner (LightSpeed Plus-U, GE Medical Systems) with multidetector-row capability. The data were obtained by using a section thickness of $0.625 \mathrm{~mm}$ and a table speed of $9 \mathrm{~mm} / \mathrm{s}(120 \mathrm{kV}, 315 \mathrm{~mA})$. Zero-degree table and gantry tilt were used. Sections in DICOM format were acquired with a $512 \times 512$ matrix. Scanning was started at the $\mathrm{C} 1$ level and continued cranially parallel to orbitomeatal line to the skull vertex during the intravenous injection of contrast material at a rate of $3.5 \mathrm{~mL} / \mathrm{s}$. 


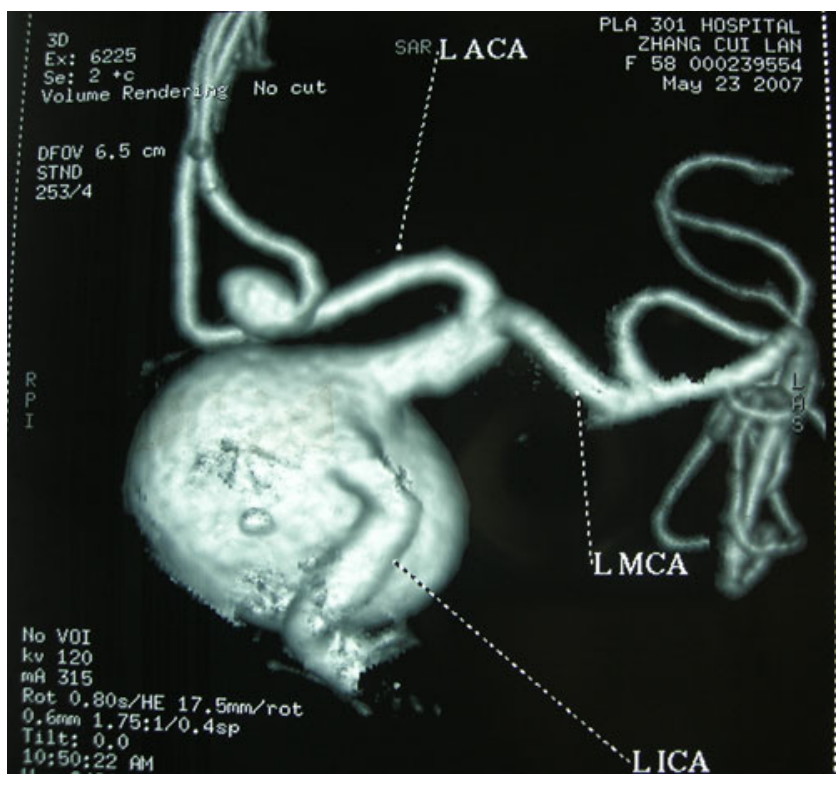

Fig. 1 CTA of an internal carotid artery paraclinoid aneurysm and an anterior communicating artery aneurysm

Rotational digital subtraction angiography

We obtained 3D datasets (LCV Plus, GE Medical Systems) from rotational series. The images with a matrix size of $512 \times 512$ pixels were acquired. All the images were immediately transferred to a workstation (Advantage Unix, GE Medical Systems) for volume rendering. A 3D reconstruction algorithm based on the algebraic reconstruction technique was used to digitally produce $3 \mathrm{D}$ digital subtraction angiography (DSA) images (Fig. 2) on the workstation, and the $3 \mathrm{D}$ project was transformed into DICOM file with a section thickness of $0.2 \mathrm{~mm}$.

\section{Reconstruction of carotid artery aneurysms}

The digital images and the respective files obtained so far could be directly imported by MIMICS software (V10.0, Belgium Materialise Company) to reconstruct a 3D project. The luminal surface of blood vessels was extracted from them in the format suitable for import by grid generators.

The main role of the MIMICS was to define the region of interest (ROI) (Fig. 3) and to extract the luminal vascular surface from the 3D raster gray-scale image obtained at the previous stage. Using the REMESH command in MIMICS, we can make mesh optimization for CFD analysis and then export the file. The procedure was fully automatic. The fluid model (blood flow) and solid model (wall) were generated. The output file could be converted into Initial Graphics Exchange Specification (IGES) file by the commercial finite element package ANSYS workbench (V11.0, ANSYS, Inc., USA) as geometry input format in most modern commercial grid generators.
Grid generator

The next stage was volume grid generation. The workbench software was used to divide fluid model into tetrahedral cells (tetrahedral grids are widely used in CFD for computational models of complex shape) (Fig. 4a). The elements chosen for the solid model were a four-node linear quadrilateral shell element and a four-node linear triangular shell element (Fig. 4b).

The number of grid nodes was chosen as a reasonable compromise between better accuracy and shorter computational time of subsequent simulations. Mesh independence was performed in order to determine the optimum number of elements. In order to gain confidence in the mesh size of fluid model, the number of elements was incrementally increased and the peak wall stress was computed. The optimum mesh size was determined once the peak stress did not increase by more than $5 \%$.

\section{Solver}

The fully coupled fluid and solid models were solved by the ANSYS workbench. The finite element method (FEM) is used to solve the governing equations in which the ALE algorithm was used. The fluid domain employs special flow-condition-based interpolation (FCBI) tetrahedral elements. We have used the formulation with large displacements and small strains in the FSI calculation available in ANSYS workbench. As iteration, we used full Newton method with a maximum of 140 iterations in each time step of 0.025 s. A sparse matrix solver based on Gaussian elimination is used for solving the system. The relative tolerance for all degrees of freedom was set to 0.001 . The force data calculated firstly in the CFX software were transferred via the workbench software to ANSYS soft-

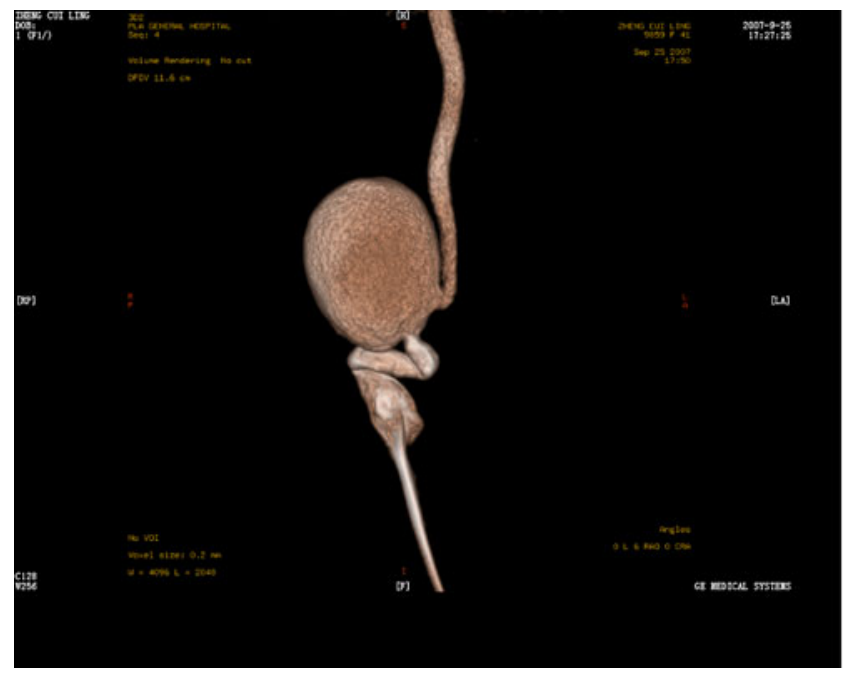

Fig. 2 3D-DSA image of a cervical internal carotid artery aneurysm 

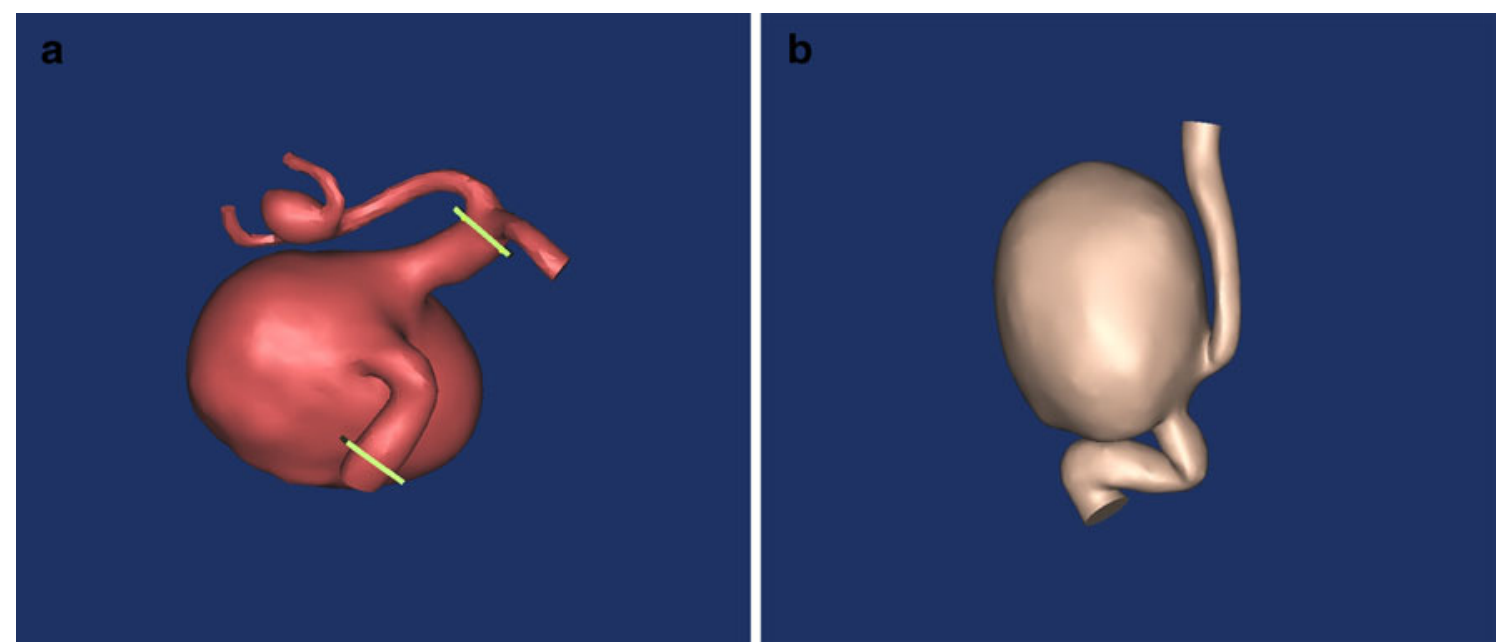

Fig. 3 Region of interest was defined (a CTA; b 3D-DSA) in MIMICS software

ware, and after receiving the force data, total mesh displacement data were calculated in ANSYS software. Then, the mesh displacement data from the ANSYS software were transferred via the workbench software to CFX software. When all the iterations were converged, the results could be visualized in CFX-post.

The workstation used is based on an Intel Xeon(TM) dual core 64 bits processor of $3.0 \mathrm{GHz}$ clock speed, 2.0 GB RAM memory and running on Microsoft Windows XP operating system. The simulation time for one FSI case based on three consecutive pulsatile flow cycles employing 150 time iterations was approximately 12 Central Processing Unit (CPU) hours.

\section{Results}

As seen in Fig. 5, the WSS, wall total pressure, blood streamline, VMS and total mesh displacement are presented in the computation. The aneurysm wall served as an obstacle decelerating the entering stream (diminishing its velocity) and deflecting it along the wall. This was accompanied by a persisting local fluid-induced WSS maximum in the area hit by the stream. The distribution of total pressure is similar with WSS. The distribution of VMS (Fig. 5e) is complex and completely different with WSS (Fig. 5b), but the VMS distribution is nearly proportion to the total mesh displacement distribution (Fig. 5f).

Figure 6 shows data from a patient with a cervical internal carotid artery aneurysm and illustrates the computational results as Fig. 5.

\section{Discussion}

The method for anatomic reconstruction of cerebral vessels should work for a broad spectrum of medical image obtained from all currently available modalities of cerebral
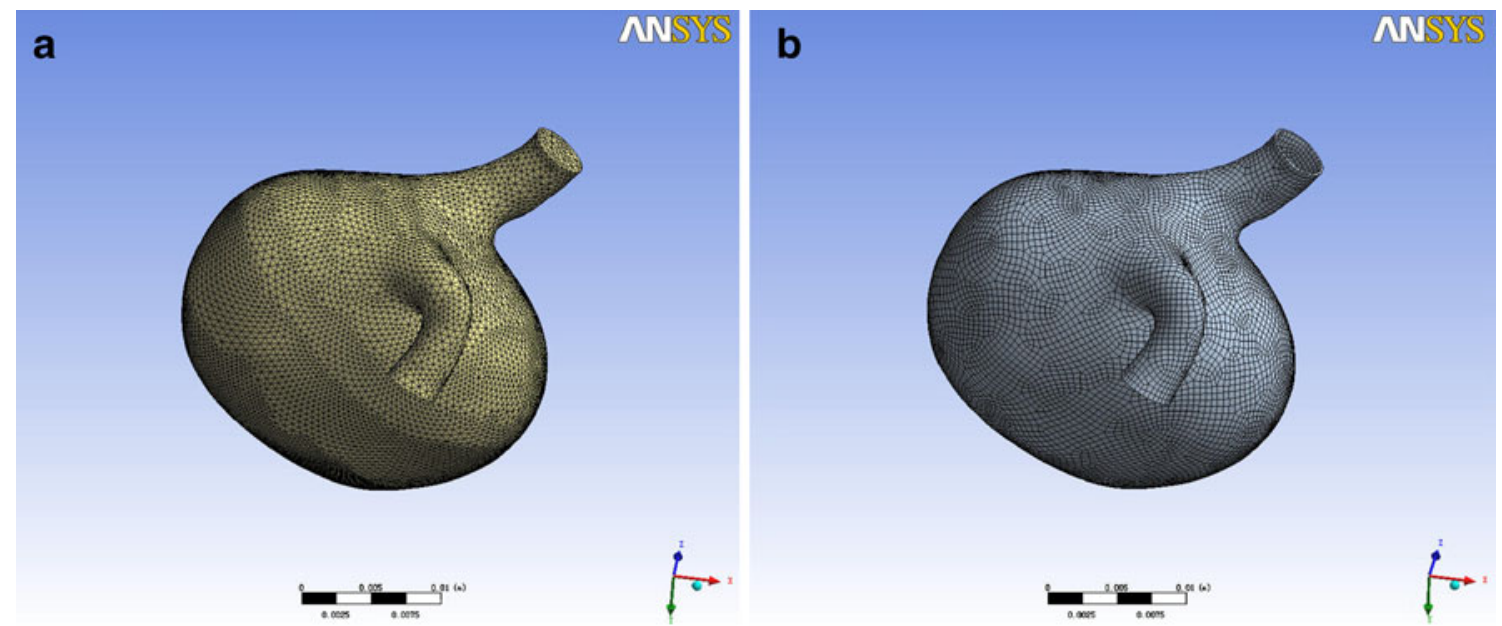

Fig. 4 Grid of fluid model (a) and solid model (b) in WORKBENCH software 

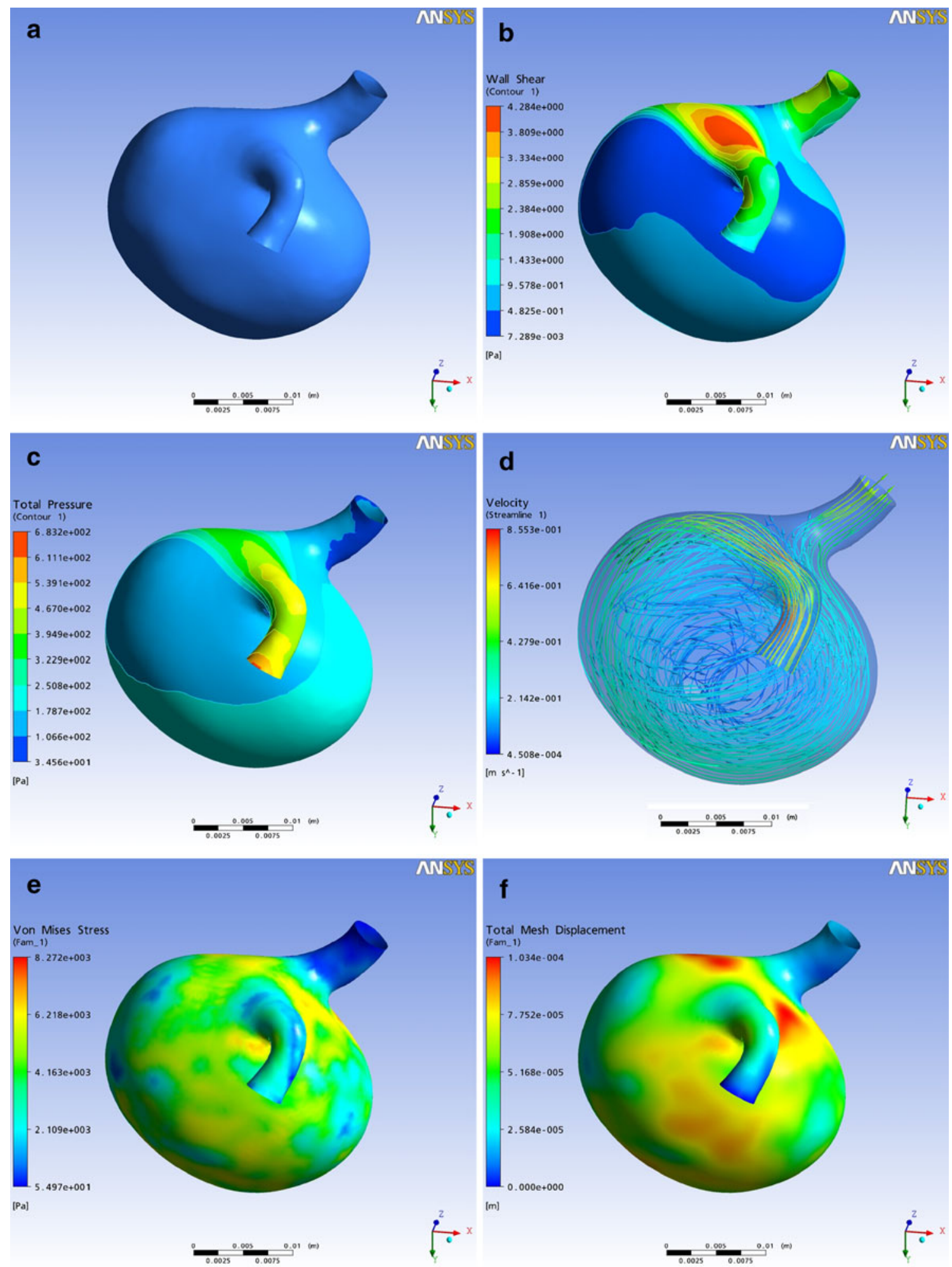

Fig. 5 Computations for the aneurysm model with CTA image. a The image of the model before deformation. $\mathbf{b}$ The contour of the WSS of the model after deformation. The red region means high stress, the blue region means low stress. c The contour of the wall's total pressure of the model after deformation. The yellow region means high pressure, the blue region means low pressure. d The streamline of the model after deformation; most of the blood flow into the aneurysm

and then flow out of the aneurysm after a couple of vortices were developed. e The image of VMS after deformation. The yellow region means high stress, the blue region means low stress. f The image of the wall's total mesh displacement after deformation. The red region means high mesh displacement, the blue region means low mesh displacement 

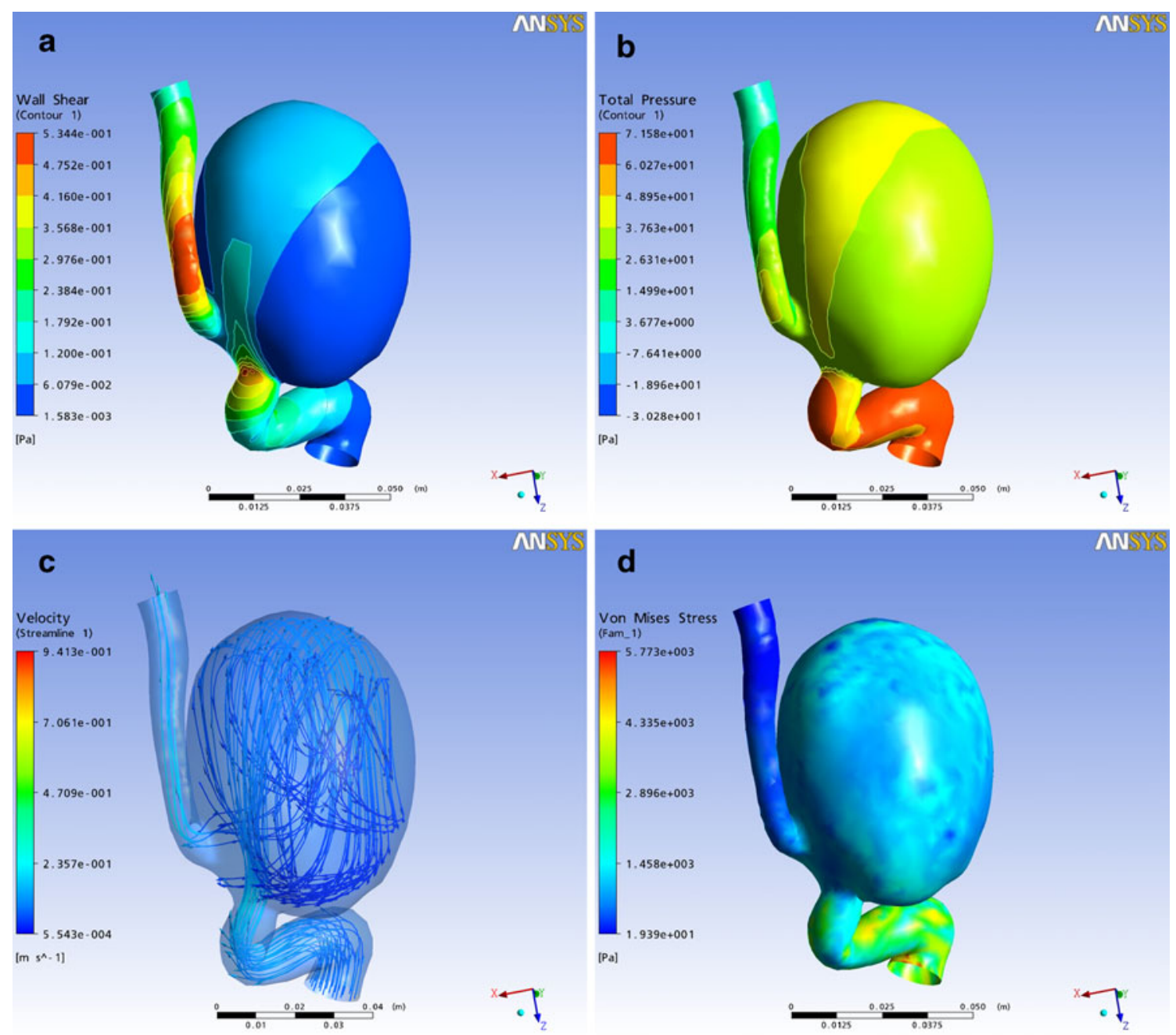

Fig. 6 Computations for the aneurysm model with 3D-DSA image. a The contour of the WSS of the model after deformation. The red region means high stress, the blue region means low stress. b The contour of the wall's total pressure of the model after deformation. The yellow region means high pressure, the blue region means low

pressure. c The streamline of the model after deformation. Most of the blood flow into the aneurysm and then flow out of the aneurysm after a couple of vortices were developed. $\mathbf{d}$ The image of wall VMS after deformation. The yellow region means high stress, the blue region means low stress

angiography such as noninvasive screening CTA, which can sometimes replace 3D-DSA in diagnosing intracranial aneurysms [1]. Therefore, our approach was general. It worked with any 3D image data in DICOM format as the source of original information, and it could produce grid output for virtually any commercial CFD software.

The 3D image data in DICOM format could be imported by MIMICS software. Therefore, in MIMICS software we directly manipulated the original source output of the angiography machines without any modifications and used it to extract the luminal surface. This approach substantially contributed to the simplicity and stability of operations and minimized possible errors of image interpolation.

Direct 3D segmentation in MIMICS software is simple and fully automatic and requires minimal effort. The average time of image segmentation from DSAs or CTAs into luminal surface meshes (Standard Template Library files) is about $40 \mathrm{~min}$. The resulting surface meshes from densely sampled high-spatial-resolution data (e.g., DSA images) are of high quality. They need no or only minor manipulations in the mesh generator before volume mesh generation (which is an automatic procedure). Sparsely sampled objects (e.g., magnetic resonance angiography images) have lower spatial resolution and therefore result in a lower quality surface mesh. These need more effort from the examiner to remove image artifacts and to repair and smooth the surface mesh before tetrahedral mesh generation [11]. Future advances in angiographic technology to further improve DSA and CTA image quality will certainly simplify and improve the accuracy of luminal surface segmentation.

3D images are manually optimized to define the vascular luminal surface before volume grid generation. Therefore, 
correction for the penumbra effect (unsharpness of edge determination due to diminished contrast resolution as the luminal vascular boundary is approached) is operator dependent with this method.

Our experience with segmentation in MIMICS software was that combined manual segmentation and automatic segmentation were very important. Sometimes false communications (e.g., between small arteries and an aneurysm) may arise, we can use manual segmentation to eliminate such false connections, and in most times automatic segmentation could be simple and saves much time.

The choice of ROI primarily depends on the examining physician. How far is the inflow and outflow boundaries depends on our knowledge of incompressible fluid dynamics, so we should solicit help from a fluid dynamics specialist to learn about the possible strong fluid dynamic effects proximal and distal to the aneurysm; such effects are due to factors such as severe arterial curvature, which may cause large secondary flows. The inflow boundary should be set within unidirectional fluid flow without any vortices or secondary flows.

Special attention should be paid to cutting out ROI and downsizing the image. Minimization of the ROI is important to decrease computational time (for both grid generation and computation itself), making it easy to finish the patientspecific image processing and computational procedure in a short time as soon as possible. Nevertheless, these considerations about the possible influence of inflow-outflow boundaries on the results should be always kept in mind.

Applications of CFD as an assisting tool for studying patient-specific hemodynamics are becoming more practical. Several clinical applications of CFD in cerebral aneurysms of real patients have been described [2, 13, 14]. Detection of the rupture area of intracranial aneurysms before surgery is important for every neurosurgeon. Although somewhat simplistic modeling that assumes rigid vessel walls may be promising for this purpose in some cases because of the relatively simple CFD technique and short computational time. However, the FSI model is more realistic than the rigid model. In our experience, when the size of an aneurysm is less than $1 \mathrm{~cm}$, the whole computational analysis lasted an average of approximately $8 \mathrm{~h}$, which makes it possible to treat patients the same day as an acute hemorrhage. Because most ruptured aneurysms are small aneurysms, this method could provide CFD results before surgery.

WSS is a flow-induced stress that can be described as the frictional force of viscous blood. Recent studies have indicated the involvement of WSS in the formation of saccular cerebral aneurysms [9]. Hassan et al. [8] found that the ruptured area of the aneurysms geographically corresponded to the area in which the bloodstream entering the aneurysm hit its wall. This area also corresponded to relatively high pressure- and fluid-induced WSS in the aneurysms. This finding would not have been possible without angiographic or operative confirmation. In general, when a blood jet impinges on an aneurysm wall, there is a stagnation point at which the pressure is maximal but WSS is zero. Around this localized high-pressure spot is a large area of high WSS caused by the bloodstream turning along the aneurysmal wall; this is where rupture happens. Tateshima et al. [17] found that the bleb of an aneurysm is exposed to a shear stress higher than that of any other measured point. WSS is a dynamic frictional force induced by a viscous fluid moving along a surface of solid material. The endothelium regulates local vascular tone by releasing vasodilator and vasoconstrictor substances. It is sensitive to changes in oscillating WSS, which has a stronger biologic influence on vessels by impinging on various endothelial functions than direct mechanical force $[10,15]$. Increased WSS is regarded as a major factor in the development and growth of cerebral aneurysms. Increased WSS caused by increased flow velocity stimulates the release of endothelium-derived nitrous oxide, which is known as a strong vasodilator and also is a potential factor in arterial wall degeneration [6]. Therefore, a local increase in WSS may cause local dilatation and degeneration of arterial walls.

The von Mises criterion is a formula for calculating whether the stress combination at a given point will cause failure. If the VMS exceeds the yield stress, then the material is considered to be at the failure condition. So the VMS may be an idea parameter to predict the rupture of aneurysms. No report about the use of VMS in intracranial aneurysms had been found; however, VMS has been studied as a potential predictor of abdominal aortic aneurysm (AAA) rupture. The VMS acting on the wall of an aneurysm are highly dependent on the shape of the specific AAA [21]. Therefore, AAAs with equivalent diameters and pressures could have largely different actual stress distributions. It is clear that, like the "maximum diameter criterion", the Law of Laplace cannot effectively describe an aneurysm's risk of rupture on a patient-specific basis. More recently, the use of peak wall stress as a potential predictor of AAA rupture was explored [19]. Fillinger et al. [4] found that the peak wall stress for AAAs which either ruptured or were symptomatic was significantly greater than that for electively repaired AAAs. In a subsequent study [5], this same group concluded that peak wall stress is a superior measure than maximum diameter for predicting patients with an unfavorable outcome. A more recent study found similar results while also showing that the location of AAA rupture correlated with the location of peak wall stress [20] In addition, DiMartino et al. [3] have recently shown that the strength of an AAA wall from ruptured AAAs is significantly less than that for electively repaired AAAs. Taken alone, much like the peak 
wall stress correlation to rupture risk, this data might suggest that AAA wall strength on its own is predictive of an aneurysm rupture. However, based on the principles of material failure, consideration of neither AAA wall stress nor wall strength alone is sufficient to assess rupture potential, but rather knowledge of both is necessary.

Model validation is an essential component of CFD simulation work. Comparing fluid dynamic data from laboratory and clinical studies with simulations should not be neglected. This is especially important for patientspecific analysis in which faithful representation of the geometry is required to achieve meaningful results. We had taken a model validation by measuring the velocity of aneurysm and parent artery with intraoperative microvascular Doppler ultrasonography; these results will be published in our other papers.

Any simulation study is based on a number of simplifying assumptions such as considering blood as a Newtonian fluid, neglecting effect of gravity and position, and others. The validity of these assumptions seems to be of secondary importance compared with the influence of the geometry; these are the most important factors for predicting possible aneurysmal growth and rupture.

Furthermore, it is well known that the aneurysm wall is significantly thinner than that of the connecting branch vessels. In the future, we plan to increase the length of the inlet and outlet branches to minimize their effect on the computed solution and also include a variable arterial wall thickness in the simulations.

\section{Conclusion}

We sought a practical method that neurosurgeons or neuroendovascular surgeons could use for CFD analysis. In this numerical simulation model, the geometry of patient-specific aneurysms has been simplified in order to decrease the computational time; however, the essential elastic feature of vessel and aneurysm has been considered through the FSI. Patient-specific computer modeling, combined with information from other imaging modalities, may provide important insight into flow dynamics before and after surgical or endovascular treatment. Future analyses will address the effects of wall pressure and wall material non-uniformities on the shear stress development.

Acknowledgement This work was supported by the Beijing Natural Scientific Foundation of China (Grant No: 7093133).

Open Access This article is distributed under the terms of the Creative Commons Attribution Noncommercial License which permits any noncommercial use, distribution, and reproduction in any medium, provided the original author(s) and source are credited.

\section{References}

1. Anderson GB, Steinke DE, Petruk KC, Ashforth R, Findlay JM (1999) Computed tomographic angiography versus digital subtraction angiography for the diagnosis and early treatment of ruptured intracranial aneurysms. Neurosurgery 45:13151322

2. Cebral JR, Castro MA, Burgess JE, Pergolizzi RS, Sheridan MJ, Putmana CM (2005) Characterization of cerebral aneurysms for assessing risk of rupture by using patient-specific computational hemodynamics models. AJNR Am J Neuroradiol 26:2550-2559

3. DiMartino ES, Bohra A, VandeGeest JP, Gupta N, Makaroun M, Vorp D (2006) Biomechanical properties of ruptured versus electively repaired abdominal aortic aneurysm wall tissue. J Vasc Surg 43:570-576

4. Fillinger MF, Raghavan ML, Marra SP, Cronenwett JL (2002) In vivo analysis of mechanical wall stress and abdominal aortic aneurysm rupture risk. J Vasc Surg 36:589-597

5. Fillinger MF, Marra SP, Raghavan ML, Kennedy F (2003) Prediction of rupture risk in abdominal aortic aneurysm during observation: wall stress versus diameter. J Vasc Surg 37:724-732

6. Guzman RJ, Abe K, Zarins CK (1997) Flow-induced arterial enlargement is inhibited by suppression of nitric oxide synthase activity in vivo. Surgery 122:273-280

7. Hassan T, Ezura M, Timofeev EV, Tominagac T, Saitob T, Takahashia A, Takayamab K, Yoshimotoc T (2004) Computational simulation of therapeutic parent artery occlusion to treat giant vertebrobasilar aneurysm. AJNR Am J Neuroradiol 25:63-68

8. Hassana T, Timofeevd EV, Saitoc T, Shimizue H, Ezuraa M, Tominagab T, Takahashia A, Takayamad K (2004) Computational replicas: anatomic reconstructions of cerebral vessels as volume numerical grids at three-dimensional angiography. Am J Neuroradiol 25:1356-1365

9. Kondo S, Hashimoto N, Kikuchi H, Hazama F, Nagata I, Kataoka H (1997) Cerebral aneurysms arising at nonbranching sites. An experimental study. Stroke 28:398-403

10. Luscher TF, Tanner FC (1993) Endothelial regulation of vascular tone and growth. Am J Hypertens 6:283S-293S

11. Moore JA, Steinman DA, Ethier CR (1998) Computational blood flow modeling errors associated with reconstructing finite element models from magnetic resonance imaging. J Biomech 31:179-184

12. Satoh T, Onoda K, Tsuchimoto S (2003) Visualization of intraaneurysmal flow patterns with transluminal flow images of $3 \mathrm{D}$ MR angiograms in conjunction with aneurysmal configurations. AJNR Am J Neuroradiol 24:1436-1445

13. Shojima M, Oshima M, Takagi K, Torii R, Hayakawa M, Katada K, Morita A, Kirino T (2004) Magnitude and role of wall shear stress on cerebral aneurysm. Computational fluid dynamic study of 20 middle cerebral artery aneurysms. Stroke 35:2500-2505

14. Shojima M, Oshima M, Takagi K, Torii R, Nagata K, Shirouzu I, Morita A, Kirino T (2005) Role of the bloodstream impacting force and the local pressure elevation in the rupture of cerebral aneurysms. Stroke 36:1933-1938

15. Stamler JS (1994) Redox signaling: nitrosylation and related target interactions of nitrous oxide. Cell 78:931-936

16. Steinman DA, Milner JS, Norley CJ, Lowniea SP, Holdswortha DW (2003) Image-based computational simulation of flow dynamics in a giant intracranial aneurysm. AJNR Am J Neuroradiol 24:559-566

17. Tateshima S, Murayama Y, Villablanca JP, Morino T, Takahashi H, Yamauchi T, Tanishita K, Vinuela F (2003) In vitro measurement of fluid-induced wall shear stress in unruptured cerebral aneurysms harboring blebs. Stroke 34:187-192

18. Valencia A, Baeza F (2009) Numerical simulation of fluidstructure interaction in stenotic arteries considering two layer 
nonlinear anisotropic structural model. Int Commun Heat Mass Transf 36:137-142

19. Vallabhaneni SR, Gilling-Smith GL, How TV, Carter SD, Brennan JA, Harris PL (2004) Heterogeneity of tensile strength and matrix metalloproteinase activity in the wall of abdominal aortic aneurysms. J Endovasc Ther 11:494-502

20. Venkatasubramaniam AK, Fagan MJ, Mehta T, Mylankal K, Ray B, Kuhan G, Chetter I, McCollum P (2004) A comparative study of aortic wall stress using finite element analysis for ruptured and non-ruptured abdominal aortic aneurysms. Eur J Vasc Endovasc Surg 28:168-176

21. Vorp DA, Raghavan ML, Webster MW, Pittsburgh P (1998) Mechanical wall stress in abdominal aortic aneurysm: influence of diameter and asymmetry. J Vasc Surg 27:632-639

\section{Comments}

\section{Nobutaka Kawahara, Yokohama, Japan}

Analysis of cerebral aneurysm by computer fluid dynamics simulation is an emerging field of research, which may be partly due to availability of various commercial software for workstations. Such analysis would provide insight into aneurysmal formation, growth, and rupture. In particular, a recent study suggested that lower, not higher, wall shear stress would induce endothelial degeneration, which may lead to growth of aneurysm.

In this paper, the authors presented a simple method to combine fluid and wall (solid) models to assess wall shear stress. Most of the previous studies assume that the arterial or aneurysmal wall is rigid, not compliant, so that the wall movement is neglected. This is largely due to unknown factors and properties of individual arterial wall (young elastic wall vs. aged atherosclerotic wall). In this manuscript, the authors have made several assumptions on the wall properties and incorporated fluid-solid interaction in the simulated model. This approach is certainly an advancement in this field; however, we should keep in mind that the wall properties would change from time to time when the wall is extended during pulsation, which is not considered in the current model. Further theoretical progress is expected to solve this issue.

Aneurysmal computer fluid and/or solid dynamic simulation would certainly provide us new insight on the natural history of aneurysms. Such approach would in turn lead to more valid assessments of treatment strategies.

Gerasimos Baltsavias and Anton Valavanis, Zurich, Switzerland

$\mathrm{Xu}$ bai-nan et al. in their paper present a method to reconstruct cerebral aneurysms as 3D numerical grids aiming to the accurate duplication of the aneurysm geometry and providing computer simulations of the blood flow. In addition, they tried to develop a method to analyze the interaction of blood flow with an elastic rather than rigid arterial wall model. As they state, when the size of an aneurysm is less than $1 \mathrm{~cm}$, the whole computational analysis lasted on average approximately $8 \mathrm{~h}$.

We certainly agree that the development of appropriate computational qualitative and quantitative simulations of flow dynamics and custom models for intracranial aneurysms in combination with further understanding of their biology may offer considerable benefits in the future management of this disease. This is becoming more evident if we consider the increasing number of unruptured aneurysms that we face in daily practice. The depiction of relevant parameters in individual aneurysms will enhance our ability to predict aneurysm rupture.

Computer simulation may help us in advancing our understanding of hemodynamic stress and the pathophysiology of brain vascular disease. Further steps are certainly necessary and soon it will be possible to add more pieces in the puzzle, as patient-specific wall motion through high-resolution noninvasive imaging techniques, details about wall anomalies, etc.

In this process there are two points (with a common actual background) that we should emphasize.

The first point is related to the rapid progress of our capacity to develop new scientific fields and tools, the increasing subspecialization of knowledge, and our objectively limited individual ability to stay up to date with this progress. There is no doubt that for the vast majority of clinicians, several concepts, methods and tools described in the paper are unknown and more important; the exact appreciation of the significance of some assumptions inherent to such processes is difficult. This emphasizes the need for substantial and effective interdisciplinary communication and leads us to the second point.

As the authors correctly pointed out, any simulation study is based on a number of simplifying assumptions, such as considering blood as a Newtonian fluid, neglecting effect of gravity and position, and others. Moreover, several steps of the simulation process, as once again the authors noticed, are subject to several artificial limitations or represent a result of compromise between several factors (as number of grid nodes) or are operator dependent (as the correction for the penumbra effect or the choice of ROI). These are some of the weak points of the method as in any scientific method and perhaps not the most important. Actually, I do not agree that the influence of geometry is the most important factor for predicting possible aneurysmal growth and rupture, as the authors state. In the kingdom of biology, geometry cannot be the king. It can be a significant or very crucial factor especially in some cases, but clinicians should not underestimate the fact that we are talking about tissues, cells, and molecules and not just about tubes, Newtonian or non-Newtonian fluids, or mechanics. An artery wall is a living, layered, continuously pulsating, and continuously remodeled tissue, interacting with the flowing blood and its molecules but also with the surrounding CSF, arachnoid chordae and membranes, brain parenchyma, bone and dura matter, carrying or not an inherited defect and influenced or not by systemic diseases (as hypertension) to mention just few of the aspects of this issue and neglect the diseased aneurysmatic wall. This wall is an innervated tissue with still many unknown aspects and functions. I am wondering how such a complex reality and multifactorial interaction can be represented in a simplifying computer model. Any progress, either in vitro experiments or in computer simulations or other, is welcomed with the caveat that we bear in mind the general frame of the reality we are dealing with.

\section{Ralf Alfons Kockro, Zurich, Switzerland}

Interdisciplinary collaboration may lead to valuable findings and concepts as it is magnificently demonstrated by this paper. The authors report on the use of algorithms of CFD in particular, considering the elasticity of aneurysm walls to simulate and threedimensionally visualize blood flow, shear stress, and aneurysm wall pressure. Two patient-specific examples of ICA aneurysms are illustrated. The results illustrate that computer modeling based on patient-specific vascular imaging data has the potential to provide important and accurate information about flow dynamics and structural properties of vessel walls before and after surgical or endovascular treatment especially in cases of unruptured aneurysms. The understanding of flow dynamics is likely to be a decisive factor in the prediction of aneurysm growth, deformation and chance of rupture, and this information is enormously important for the establishment of timelines and strategies of treatment planning. Structural imaging and computational physics complement and enhance each other and offer the potential to simulate and predict tissue deformation and mechanical behavior and therefore significantly push the boundaries of current static structural visualization. 